\title{
MODELING OF ROTATING BLADED DISKS BY USING CONSERVATIVE MODAL ANALYSIS
}

\author{
D. Rychecký ${ }^{*}$, M. Hajžman ${ }^{* *}$, V. Zeman ${ }^{* * *}$
}

\begin{abstract}
The efficient method usable for the vibrational analysis of interacting flexible bodies is presented in this paper. It is motivated by the research of blades interaction in the framework of turbine bladed disks. The approach is based on the exporting system matrices, eigenfrequencies and eigenmodes for one blade from finite element software and assembling mathematical model in MATLAB system in an in-house code. The implemented code enables composing bladed disk model and more effective solution of dynamic problems including contact phenomena, prestress, etc.
\end{abstract}

Keywords: dynamics, contact, friction, blades, prestress

\section{Introduction}

Solution of real industrial problems is often computationally limited by time consuming procedures. Although hardware is getting better and better, approaches for reduction of solution time are still necessary. The method presented in this paper is motivated by the research of blades interaction in the framework of turbine bladed disks (Půst and Pešek, 2010). The time effective prediction of the bladed disk vibrations allows to optimize bladed disk's parameters. This fact leads to successive blade vibration suppression more precisely with higher efficiency (Petrov and Ewins, 2006).

This paper is focused on one of the possible approaches for modeling bladed disks composed by general geometry of the blade including constant rotation. The purpose of the paper is the description of the method for exporting basic data from a finite element solver (ANSYS in this case) and assembling of the mathematical model of the bladed disk with prestress in MATLAB system. The methodology presented in this paper is suitable for many applications.

\section{Mathematical model}

General blade geometry usually meshed by 8 -node 3D finite elements is considered. The generalized coordinate vector of nodal displacements of $i$-th node is composed of three translations $\mathbf{q}=\left[u_{i}, v_{i}, w_{i}\right]^{T}$ The finite element model of one rotating flexible blade includes all effects of stationary rotation (defined by constant angular velocity $\omega_{0}$ ) and can be written in the form

$$
\mathbf{M} \ddot{\mathbf{q}}+\left(\mathbf{B}+\omega_{0} \mathbf{G}\right) \dot{\mathbf{q}}+\left(\mathbf{K}_{S}-\omega_{0}^{2} \mathbf{K}_{d}+\omega_{0}^{2} \mathbf{K}_{\omega}\right) \mathbf{q}=\omega_{0}^{2} \mathbf{f}_{\omega}+\mathbf{f}(\mathbf{q}, \dot{\mathbf{q}}, t),
$$

where symmetric matrices $\mathbf{M}, \mathbf{B}, \mathbf{K}_{S}, \omega_{0}^{2} \mathbf{K}_{d}, \omega_{0}^{2} \mathbf{K}_{\omega}$ are mass, material damping, static stiffness, spin softening and centrifugal stiffening matrices. Matrix $\omega_{0} \mathbf{G}$ is a skew symmetric matrix of gyroscopic effects. Constant centrifugal forces are expressed by vector $\omega_{0}^{2} \mathbf{f}_{\omega}$. Right-hand side vector $\mathbf{f}(\mathbf{q}, \dot{\mathbf{q}}, t)$ includes timevariant excitation forces, normal contact forces and tangential (friction) contact forces.

* Ing. Drahomír Rychecký: Department of Mechanics, University of West Bohemia, Univerzitní 8, 30100 Plzeň; CZ, rychecky@kme.zcu.cz

** Ing. Michal Hajžman, Ph.D.: Department of Mechanics, University of West Bohemia, Univerzitní 8, 30100 Plzeň; CZ, mhajzman@kme.zcu.cz

*** Prof. Ing. Vladimír Zeman, DrSc.: Department of Mechanics, University of West Bohemia, Univerzitní 8, 30100 Plzeň; CZ, zemanv@kme.zcu.cz 
The presented method is based on the modal analysis of a conservative model of one undamped blade without gyroscopic effects

$$
\mathbf{M} \ddot{\mathbf{q}}+\mathbf{K}_{S} \mathbf{q}=\mathbf{0},
$$

which gives matrices of eigenmodes $\mathbf{V}$ and eigenvalues $\boldsymbol{\Lambda}$ normalized by $\mathbf{M}$-norm. These matrices are exported from ANSYS software. Matrices $\omega_{0} \mathbf{G}, \omega_{0}^{2} \mathbf{K}_{d}, \omega_{0}^{2} \mathbf{K}_{\omega}$ and vector $\omega_{0}^{2} \mathbf{f}_{\omega}$ are also assembled and exported using the finite element solver.

The whole reduced model is then created using in-house code implemented in MATLAB system. The transformation from generalized to modal coordinates is suitable transformation allowing a modal reduction by $m$ eigenmodes. The reduction is described by left upper index $m \mathbf{q}={ }^{m} \mathbf{V x}$. After employing this transformation and left multiplication of equation (1), it yields

$$
\begin{gathered}
{ }^{m} \mathbf{V}^{T} \mathbf{M}^{m} \mathbf{V} \ddot{\mathbf{x}}+{ }^{m} \mathbf{V}^{T}\left(\mathbf{B}+\omega_{0} \mathbf{G}\right)^{m} \mathbf{V} \dot{\mathbf{x}}+ \\
+{ }^{m} \mathbf{V}^{T}\left(\mathbf{K}_{S}-\omega_{0}^{2} \mathbf{K}_{d}+\omega_{0}^{2} \mathbf{K}_{\omega}\right)^{m} \mathbf{V} \mathbf{x}=\omega_{0}^{2}{ }^{m} \mathbf{V}^{T} \mathbf{f}_{\omega}+{ }^{m} \mathbf{V}^{T} \mathbf{f}(\mathbf{x}, \dot{\mathbf{x}}, t) .
\end{gathered}
$$

Utilizing notation

$$
\begin{gathered}
\widetilde{\mathbf{B}}={ }^{m} \mathbf{V}^{T} \mathbf{B}^{m} \mathbf{V}, \quad \widetilde{\mathbf{G}}={ }^{m} \mathbf{V}^{T} \mathbf{G}^{m} \mathbf{V}, \quad \widetilde{\mathbf{K}}_{\Omega}\left(\omega_{0}\right)={ }^{m} \mathbf{V}^{T}\left(-\omega_{0}^{2} \mathbf{K}_{d}+\omega_{0}^{2} \mathbf{K}_{\omega}\right)^{m} \mathbf{V}, \\
\widetilde{\mathbf{f}}_{\omega}={ }^{m} \mathbf{V}^{T} \mathbf{f}_{\omega}, \quad \widetilde{\mathbf{f}}={ }^{m} \mathbf{V}^{T} \mathbf{f}(\mathbf{x}, \dot{\mathbf{x}}, t),
\end{gathered}
$$

mathematical model of one blade (3) can be rewritten in the form

$$
\ddot{\mathbf{x}}+\left(\widetilde{\mathbf{B}}+\omega_{0} \widetilde{\mathbf{G}}\right) \dot{\mathbf{x}}+\left(\boldsymbol{\Lambda}+\widetilde{\mathbf{K}}_{\Omega}\left(\omega_{0}\right)\right) \mathbf{x}=\omega_{0}^{2} \widetilde{\mathbf{f}}_{\omega}+\widetilde{\mathbf{f}} .
$$

Advantage of this approach are constant values of matrices $\widetilde{\mathbf{B}}, \widetilde{\mathbf{G}}$ a $\boldsymbol{\Lambda}$ and vector $\widetilde{\mathbf{f}}$ for different constant angular velocity $\omega_{0}$. Only matrix $\widetilde{\mathbf{K}}_{\Omega}\left(\omega_{0}\right)$ depends on angular velocity.

Mathematical model of a bladed disk is created by $j$ separate mathematical models (4). Elastic contact between blades is included in force vector $\widetilde{\mathbf{f}}_{i}, i=1,2, \ldots j$. We get

$$
\begin{aligned}
& \underbrace{\left[\begin{array}{c}
\widehat{\ddot{\boldsymbol{x}}}_{1} \\
\widehat{\ddot{\boldsymbol{x}}}_{2} \\
\vdots \\
\widehat{\ddot{\boldsymbol{x}}}_{j}
\end{array}\right]}_{\widehat{\hat{\boldsymbol{x}}}}+\underbrace{\left[\begin{array}{cccc}
\widetilde{\mathbf{B}}+\omega_{0} \widetilde{\mathbf{G}} & \mathbf{0} & \mathbf{0} & \mathbf{0} \\
\mathbf{0} & \widetilde{\mathbf{B}}+\omega_{0} \widetilde{\mathbf{G}} & \mathbf{0} & \mathbf{0} \\
\mathbf{0} & \mathbf{0} & \ddots & \mathbf{0} \\
\mathbf{0} & \mathbf{0} & \mathbf{0} & \widetilde{\mathbf{B}}+\omega_{0} \widetilde{\mathbf{G}}
\end{array}\right]}_{\widehat{\mathbf{B}}+\omega_{0} \widehat{\mathbf{G}}} \underbrace{\left[\begin{array}{c}
\widehat{\hat{\boldsymbol{x}}_{1}} \\
\widehat{\boldsymbol{x}_{2}} \\
\vdots \\
\widehat{\hat{\boldsymbol{x}}_{j}}
\end{array}\right]}_{\widehat{\hat{\boldsymbol{x}}}}+ \\
& +\underbrace{\left[\begin{array}{cccc}
\boldsymbol{\Lambda}+\widetilde{\mathbf{K}}_{\Omega}\left(\omega_{0}\right) & \mathbf{0} & \mathbf{0} & \mathbf{0} \\
\mathbf{0} & \boldsymbol{\Lambda}+\widetilde{\mathbf{K}}_{\Omega}\left(\omega_{0}\right) & \mathbf{0} & \mathbf{0} \\
\mathbf{0} & \mathbf{0} & \ddots & \mathbf{0} \\
\mathbf{0} & \mathbf{0} & \mathbf{0} & \boldsymbol{\Lambda}+\widetilde{\mathbf{K}}_{\Omega}\left(\omega_{0}\right)
\end{array}\right]}_{\widehat{\boldsymbol{\Lambda}}+\widehat{\mathbf{K}}_{\Omega}\left(\omega_{0}\right)} \underbrace{\left[\begin{array}{c}
\widehat{\boldsymbol{x}}_{1} \\
\widehat{\boldsymbol{x}}_{2} \\
\vdots \\
\widehat{\boldsymbol{x}}_{j}
\end{array}\right]}_{\widehat{\mathbf{x}}}=\underbrace{\left[\begin{array}{c}
\widetilde{\mathbf{f}}_{\omega} \\
\widetilde{\mathbf{f}}_{\omega} \\
\vdots \\
\widetilde{\mathbf{f}}_{\omega}
\end{array}\right]}_{\widehat{\mathbf{f}}_{\omega}}+\underbrace{\left[\begin{array}{c}
\widetilde{\mathbf{f}}_{1} \\
\widetilde{\mathbf{f}}_{2} \\
\vdots \\
\tilde{\mathbf{f}}_{j}
\end{array}\right]}_{\widehat{\mathbf{f}}} .
\end{aligned}
$$

Prestress effect is included adding stiffness matrix $\mathbf{K}_{P S}$. Non-zero stiffness parameters are on positions of contact points.

\section{Testing of the mathematical approach for modeling rotating bodies}

Testing of the presented approach for modelling rotating bodies by a conservative modal analysis was made by a simple blade geometry. The simple geometry was chosen with respect to time effective evaluation. It is shown in Fig. 1. The correct approach for the export of a single blade defined above was tested first. The blade including rotation effects was loaded by step force $F_{z}=100 \mathrm{~N}$ (for $t=0$ jumps from 0 to $100 \mathrm{~N}$ ). The same problem was solved for full model in ANSYS (black line) and modal reduced model in MATLAB (blue line). The first 20 eigenmodes (i.e. $m=20$ ) was used. To demonstrate retunig caused by gyroscopic effects, non-rotating non-damped model is involved, see Fig. 2 (left). Comparison of three different models (1. non-rotating and non-damped, 2. rotating and non-damped, 3. non-rotating and damped) is shown in Fig. 2 (right). It is possible to see retuning again and the effect of material proportional damping. 


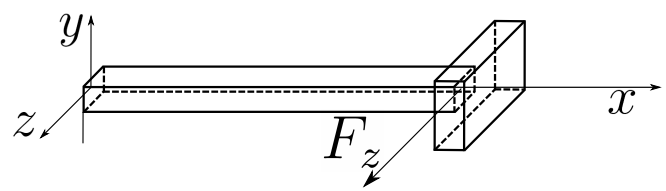

Fig. 1: Simple geometry for the testing of the proposed approach.
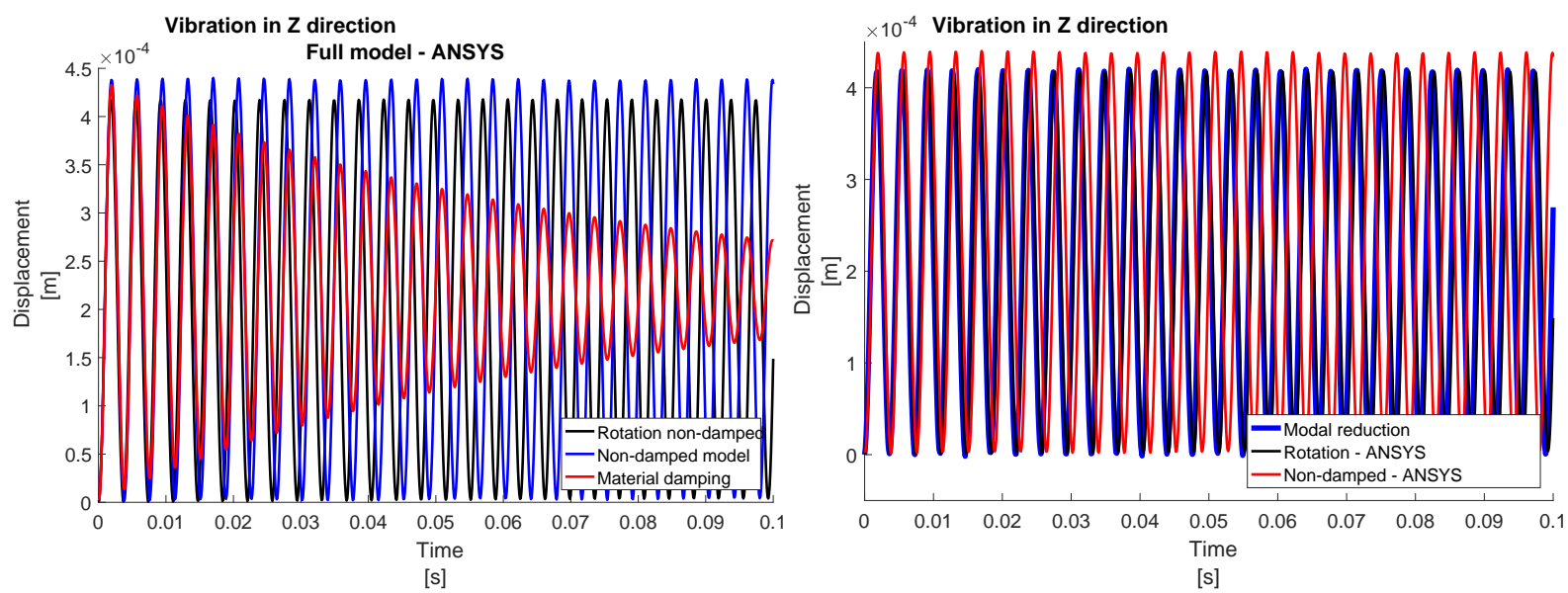

Fig. 2: Comparison of blade vibrations for different models.

\section{Modal analysis of the bladed disk}

The investigated bladed disk is composed by 100 separate blades according to presented methodology. The blades are fixed to rigid shaft. Value of the prestress is one of the important parameters affecting the eigenfrequencies of the bladed disk. Its value is derivated according to (Byrtus et al., 2010). It is 3.27 MPa for this bladed disk. The eigenvalues and eigenmodes were calculated from the conservative model of the whole bladed disk

$$
\widehat{\ddot{\boldsymbol{x}}}+\left(\widehat{\boldsymbol{\Lambda}}+\widehat{\mathbf{K}}_{\Omega}\left(\omega_{0}\right)\right) \widehat{\mathbf{x}}=\mathbf{0} .
$$

The first 5 eigenfrequencies of the bladed disk are summarized in Tab. 1. For the comparison, there are included eigenfrequencies for the bladed disk with prestress 0 MPa, 0.327 MPa, 3.27 MPa and 32.7 MPa. The eigenfrequencies are multiple frequencies for case without prestress. This is the reason to show 101st, 201st and 301st eigenfrequencies.

Tab. 1: Eigenfrequencies [Hz] of the bladed disk

\begin{tabular}{ccccc}
\hline Order & \multicolumn{4}{c}{ Prestress } \\
\hline & 0 & $0.327 \mathrm{MPa}$ & $3.27 \mathrm{MPa}$ & $32.7 \mathrm{MPa}$ \\
\hline 1 & 990.76 & 991.90 & 991.90 & 991.90 \\
\hline 2 & 990.76 & 992.76 & 992.76 & 995.20 \\
\hline 3 & 990.76 & 992.76 & 992.76 & 995.20 \\
\hline 4 & 990.76 & 995.20 & 995.20 & 998.83 \\
\hline 5 & 990.76 & 995.20 & 995.20 & 998.83 \\
\hline 101 & 2244.60 & 2244.64 & 2244.64 & 2244.64 \\
\hline 201 & 3794.00 & 3794.01 & 3794.01 & 3794.01 \\
\hline 301 & 4592.08 & 4592.36 & 4592.36 & 4592.36 \\
\hline
\end{tabular}




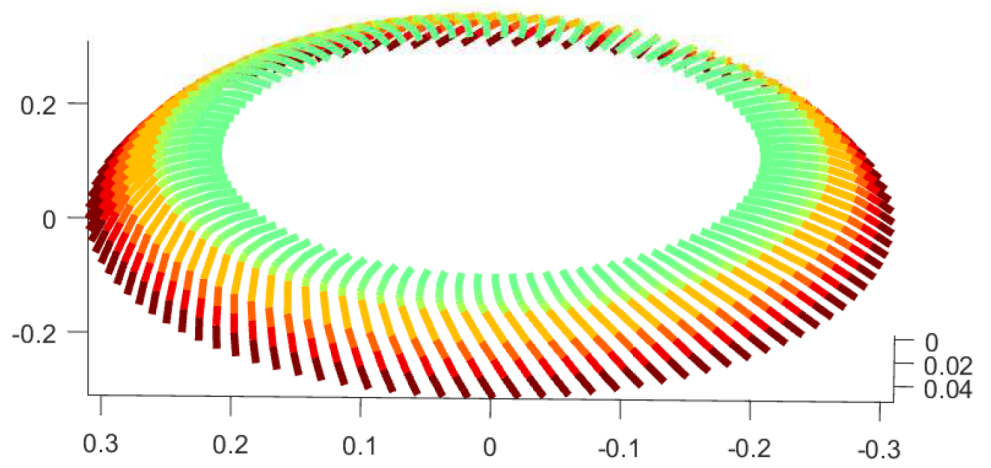

Fig. 3: The first eigenmode (991.90 Hz) of the bladed disk for prestress 3.27 MPa.

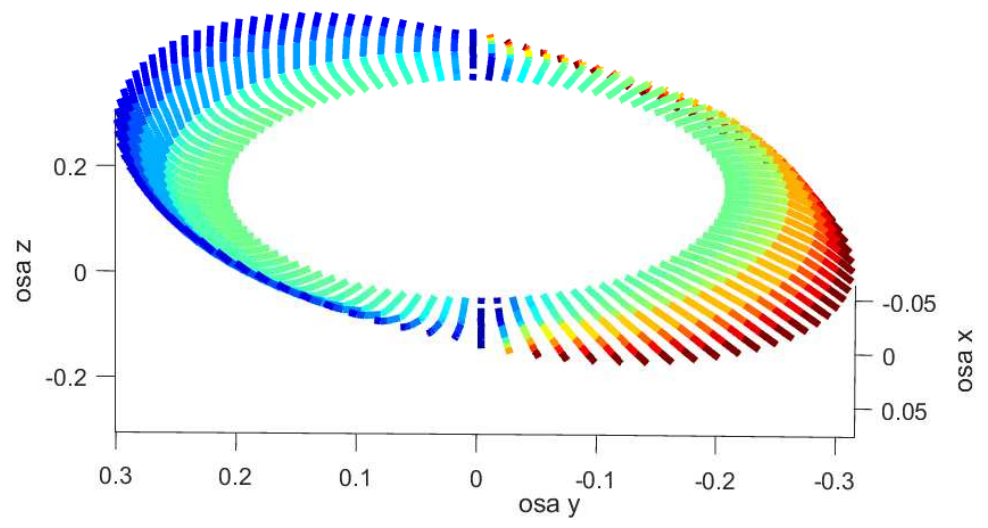

Fig. 4: The second eigenmode $(992.76 \mathrm{~Hz})$ of the bladed disk for prestress $3.27 \mathrm{MPa}$.

\section{Conclusions}

The methodology for the modelling of flexible blades vibrations including effects of rotation is briefly described in this paper. The effort is motivated by the need to solve the problems of contact dynamics of interacting blades with a real geometry. The motivation for development of this method arises from high computational costs when using commercial finite element software tools. Without suitable solution methodologies it is not possible to design, optimize and to tune proper and effective structural elements for sake of undesirable vibration suppression.

\section{Acknowledgments}

Authors were supported by the project number TE01020068 in the framework of the "Competence Centres" programme of the Technology Agency of the Czech Republic.

\section{References}

Byrtus, M., Hajžman, M. and Zeman, V. (2010) Harmonic forced vibration of two rotating blades with friction damping, Engineering Mechanics, Vol. 17, pp 187.

Petrov, E.P. and Ewins, D.J. (2006) Effects of Damping and Varying Contact Area at Blade-Disk Joints in Forced Response Analysis of Bladed Disk Assemblies, Journal of Turbomachinery, Vol 128, pp 403.

Půst, L. and Pešek, L. (2010) Non-proportional nonlinear damping in experimental bladed disk, Engineering Mechanics, Vol 17, pp 237. 